\title{
ESTUDO DA VIABILIDADE DO CAULE DE MANDIOCA (Manihot esculenta Crantz) NA ADSORÇÃO DE CORANTES
}

\author{
H. M. HEIDEMANN ${ }^{1}$, A. GILIOLI ${ }^{1}$, M. G. N. QUADRI ${ }^{*}$ \\ ${ }^{1}$ Universidade Federal de Santa Catarina, Departamento de Engenharia Química e \\ Engenharia de Alimentos \\ E-mail para contato: mara.quadri@ufsc.br
}

\begin{abstract}
RESUMO -O setor têxtil tem descartado compostos orgânicos em grandes quantidades no meio ambiente alterando o equilíbrio natural e acarretando ameaças aos ecossistemas. A remoção de corantes em efluentes industriais tem recebido enorme atenção nos últimos anos, e a adsorção é uma das técnicas empregadas com sucesso remoção de pigmentos. Devido ao alto custo de alguns adsorventes convencionais, bioadsorventes de baixo custo têm sido usados. Neste trabalho foi utilizado o talo da mandioca (Manihot esculenta Crantz), resíduo da indústria agroalimentícia, como biossorvente para a remoção dos corantes verde malaquita e azul de metileno em solução aquosa através do processo em batelada. Foram estudados os efeitos da temperatura, tempo de contato, concentração inicial do corante e tamanho de partícula na capacidade de adsorção do biomaterial. O biossorvente foi caracterizado através de FTIR, $\mathrm{pH}_{\mathrm{PCZ}}$ e MEV. Os resultados cinéticos foram bem descritos pelo modelo de pseudo-segunda ordem e as isotermas pelo modelo de Freundlich. Conclui-se deste trabalho que o caule de mandioca é um adsorvente viável, com boa capacidade de adsorção para ambos os corantes estudados.
\end{abstract}

\section{INTRODUÇÃO}

O crescimento do setor têxtil tem trazido muitos benefícios à população. Entretanto, os compostos orgânicos que são sintetizados e continuamente introduzidos em quantidades significativas no meio ambiente, podem afetar o equilíbrio natural e ameaçar os ecossistemas (Kolpin et al., 2002).

Os efluentes têxteis caracterizam-se por serem altamente coloridos devido à presença de corantes que não se fixam nas fibras durante o processo de tingimento. Os corantes são moléculas orgânicas altamente estruturadas e de difícil degradação biológica. Sua concentração é menor do que muitos outros produtos químicos encontrados nos efluentes, mas sua cor é visível até em baixas concentrações (Sarasa et al., 1998).

No Brasil, os caules e partes aéreas da mandioca (Manihot esculenta Crantz) que não são aproveitados como ração animal e na alimentação humana,são considerados resíduos, 
(Gidamis et al., 1993).A indústria de derivados de mandioca é voltada para a produção da farinha e fécula, gerando resíduos sólidos, como a casca e entrecasca, e líquidos (manipueira). Segundo Fioretto (2001), o processamento de uma tonelada por dia de raízes de mandioca em uma fecularia equivale à poluição causada por 200-300 habitantes dia. Este montante de raízes processadas em cascas de farinha corresponde a um equivalente populacional de 150250habitantes/dia. Diante disto, a produção destes derivados gera poluição não desprezível, prejudicando principalmente as populações nas cercanias das empresas (Inoue, 2008).

Este trabalho buscou avaliar a viabilidade do uso do caule da mandioca como adsorvente no tratamento de efluentes contendo os corantes azul de metileno e verde malaquita, os corantes mais usados na indústria têxtil,.

\section{MATERIAIS E MÉTODOS}

\subsection{Adsorvente e Adsorbatos}

Os corantes azul de metileno $\left(\mathrm{C}_{16} \mathrm{H}_{18} \mathrm{~N}_{3} \mathrm{SCl}\right)$ e verde malaquita $\left(\mathrm{C}_{23} \mathrm{H}_{25} \mathrm{ClN}_{2}\right)$ foram adquiridos da marca VETEC,e foram utilizados sem prévia purificação. Soluções padrão dos corantes foram preparadas na concentração de $5 \mu \mathrm{g} / \mathrm{mL}$, e, partir desta,foram feitas diluições.

O caule de mandioca foi seco ao sol, triturado, peneirado e separado por tamanhos de partículas.Os adsorventes estudados foram os de tamanhos 250, 150 e $75 \mu \mathrm{m}$.

\subsection{Caracterização do adsorvente}

O ponto de carga zero foi determinado segundo a metodologia de Faria et al., 2004.A determinação dos grupos funcionais da superfície do adsorvente seguiu o método titulométrico de Boehm (2002).

As imagens microscópicas do adsorvente foram obtidas utilizando o microscópio eletrônico de varredura JEOL JSM-6390LV, do Laboratório Central de Microscopia Eletrônica - LCME da UFSC. As amostras foram recobertas com ouro utilizando o aparelho SCD 500 da marca LEICA.

A espectroscopia na região do infravermelho foi realizada no Laboratório de Controle de Processos (Departamento de Engenharia Química e Alimentos, UFSC) em discos de $\mathrm{KBr}$, no intervalo de comprimento de onda $400-4000 \mathrm{~cm}^{-1}$ usando o espectro Shimatzu modelo IR PRESTIGE-21.

O teor de extrativos foi determinado pelo método da Technical Association of the Pulpand Paper Industry (Tapi, 1997). O teor de lignina foi determinado pelo método T249 cm-85 (Tapi, 1999), denominada lignina KLASON. Na determinação de hemicelulose e celulose foi utilizado o método de antrona segundo Dische et al. (1962). 


\subsection{Estudo cinético}

As cinéticas e as isotermas de adsorção foram conduzidas em sistema em batelada, em duplicata. Foram adicionadas $25 \mathrm{mg}$ do adsorvente em frascos erlenmeyer contendo $50 \mathrm{~mL}$ de solução dos corantes. Os frascos selados foram mantidos sob agitação em shaker.

A cinética foi realizada na temperatura de $25{ }^{\circ} \mathrm{C}$ com tempo de contato variando entre 0 e 720 minutos. As isotermas foram realizadas nas temperaturas de $25,35,45$ e $65^{\circ} \mathrm{C}$ e com as concentrações das soluções variando entre 2 e $5 \mu \mathrm{g} / \mathrm{mL}$. O efeito do pH na adsorção dos corantes também foi testado, a $25^{\circ} \mathrm{C}$, ajustando-se o $\mathrm{pH}$ de 4,0 a 11,0 pela adição de soluções $0,1 \mathrm{~mol} \mathrm{~L}^{-1}$ de $\mathrm{NaOH}$ ou $\mathrm{HCl}$.

Após os tempos de contato, as amostras foram centrifugadas e os sobrenadantes foram analisados em espectrofotômetro para determinação das concentrações finais dos corantes azul de metileno e verde malaquita nos comprimentos de onda $665 \mathrm{~nm}$ e $625 \mathrm{~nm}$, respectivamente.

As quantidades adsorvidas dos corantes foram calculadas através da Equação 1:

$$
q_{e}=\left[\left(C_{0}-C_{e}\right) \cdot V\right] / m
$$

onde, $\mathrm{q}_{\mathrm{e}}$ é a quantidade adsorvida por massa de adsorvente $(\mathrm{mg} / \mathrm{g}) ; \mathrm{C}_{0}$, concentração inicial do adsorbato $\left(\mathrm{mg} \mathrm{L}^{-1}\right) ; \mathrm{C}_{\mathrm{e}}$, concentração do adsorbato no equilíbrio $\left(\mathrm{mg} \mathrm{L}^{-1}\right) ; \mathrm{V}$, volume da solução empregado na adsorção (L); m é a massa do adsorvente utilizada (g).

Os resultados cinéticos foram ajustados aos modelos de pseudo-primeira ordem, e pseudo-segunda ordem. Os modelos de Langmuir e Freundlich foram usados para descrever o comportamento da adsorção dos adsorbatos avaliados.

Os parâmetros termodinâmicos de entalpia, entropia e energia livre de Gibbs foram calculados a partir dos dados das isotermas, utilizando as equações 2 e 3.

$$
\begin{aligned}
& \ln \left(q_{e} / C_{e}\right)=(\Delta s / R)-(\Delta H / R) \cdot(1 / T) \\
& \Delta G=\Delta H-T . \Delta S
\end{aligned}
$$

Onde $\Delta \mathrm{H}\left(\mathrm{J} \cdot \mathrm{mol}^{-1}\right)$ é a entalpia, $\Delta \mathrm{S}\left(\mathrm{J} \cdot \mathrm{mol}^{-1} \cdot \mathrm{K}^{-1}\right)$ a entropia, $\Delta \mathrm{G}\left(\mathrm{J} \cdot \mathrm{mol}^{-1}\right)$ a energia livre de Gibbs e $\mathrm{T}$ a temperatura em Kelvin.

\section{RESULTADOS E DISCUSSÕES}

\subsection{Caracterização do adsorvente}

Na Tabela 1 encontram-se os resultados da caracterização do adsorvente obtido do talo da mandioca. 
Tabela 1 - Caracterização do Adsorvente

\begin{tabular}{|c|c|}
\hline Propriedade & Valor \\
\hline Teor de umidade $(\%)$ & 13,7 \\
\hline Ponto de carga zero $\left(\mathrm{pH}_{\mathrm{pcz}}\right)$ & 6,1 \\
\hline Lignina Klason(\%) & 22 \\
\hline Extrativos $(\%)$ & 14 \\
\hline Hemicelulose $(\%)$ & 38,94 \\
\hline Celulose $(\%)$ & 37,2 \\
\hline Grupos Ácidos Totais (Eqg.g ${ }^{-1}$ ) & 0,0696 \\
\hline Grupos Lactonas (Eqg.g $\left.{ }^{-1}\right)$ & 0,02324 \\
\hline Grupos Carboxílicos (Eqg.g $\left.{ }^{-1}\right)$ & 0,01826 \\
\hline Grupos Fenólicos (Eqg.g $\left.{ }^{-1}\right)$ & 0,0281 \\
\hline
\end{tabular}

$\mathrm{O} \mathrm{pH}_{\mathrm{PCZ}}$ indica o $\mathrm{pH}$ no qual o balanço entre as cargas positivas e negativas presentes no material é nulo, ou seja as quantidades de cargas positivas $\left(\mathrm{H}^{+}\right)$e negativas $\left(\mathrm{OH}^{-}\right)$se igualam.Desta forma o adsorvente possui carga superficial negativa em valores de $\mathrm{pH}$ acima de 6,1; favorecendo a adsorção dos corantes catiônicos estudados. Os grupos ácidos presentes em maior quantidade na superfície do adsorvente são os fenólicos, seguido pelas lactonas e os ácidos carboxílicos.

As imagens obtidas por Microscopia Eletrônica de Varredura (MEV) mostradas na Figura 1 mostram o perfil estrutural do adsorvente estudado. Segundo Maieves (2010) as estruturas arredondadas são grânulos de amido, alguns visivelmente envoltos por material peptídico e celulósico.
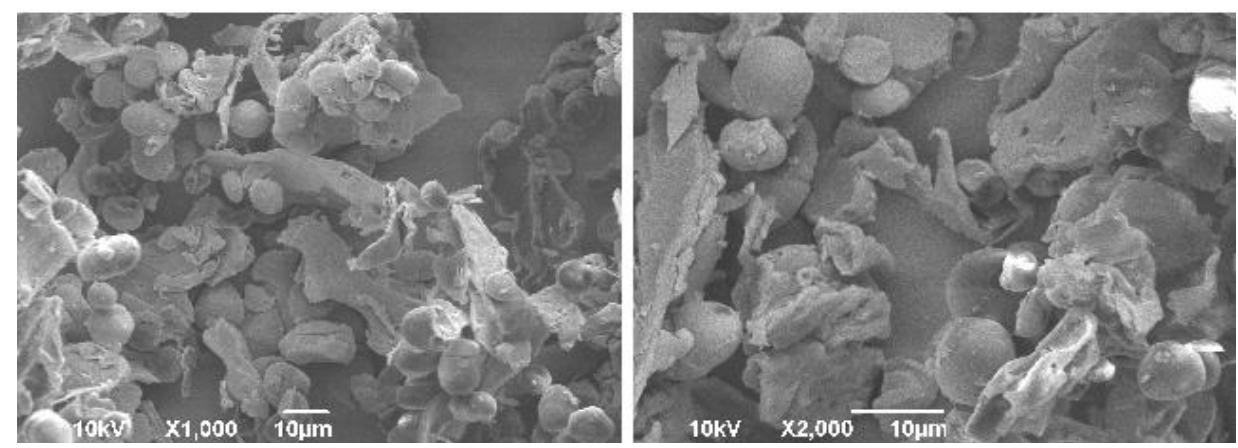

Figura 1. Microscopia Eletrônica de Varredura do talo de mandioca moído. aumento a) 1000x ; b) 2000x.

O espectro de infravermelho do adsorvente (Figura 2) apresenta uma banda de absorção larga e intensa em $3413 \mathrm{~cm}^{-1}$ corresponde a vibrações dos grupos $\mathrm{O}-\mathrm{H}$ e $\mathrm{N}-\mathrm{H}$, que neste tipo de material é atribuído a celulose, pectina, hemicelulose, lignina e água absorvida; uma banda em $2930 \mathrm{~cm}^{-1}$ pode ser atribuída ao estiramento $\mathrm{C}-\mathrm{H}$ das vibrações dos grupos 
metileno, metilo e grupos metóxi. A presença das bandas em $1735 \mathrm{~cm}^{-1}$ e $1641 \mathrm{~cm}^{-1}$ no espectro indicam as bandas de estiramento de grupos carbonila de éster e de íon carboxilato (COO-), respectivamente (Perez-marin et al., 2007).

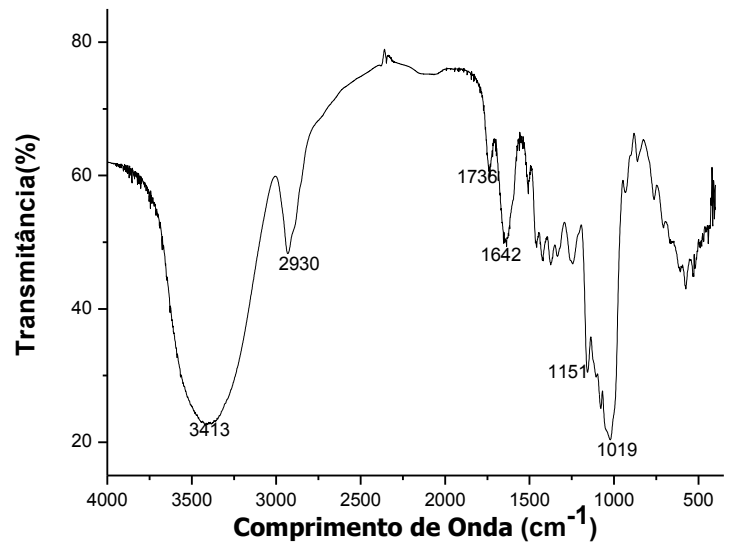

Figura2. Espectro de Infravermelho do caule da mandioca

\subsection{Cinética de adsorção}

A adsorção do azul de metileno e verde malaquita pelo caule da mandioca mostrou um processo rápido, com equilíbrio relativamente rápido(360 mim para ambos os corantes).As cinéticas foram melhor ajustadas pelo modelo de pseudo-segunda ordem, com coeficientes de determinação altos, e capacidade de adsorção próximas às teóricas. Os parâmetros cinéticos de pseudo-segunda ordem para o Azul de Metileno e Verde de Malaquita estão na Tabela 2.

Tabela2-Parâmetros cinéticos de Pseudo-segunda ordem para os corantes Azul de Metileno e verde malaquita em diferentes tamanhos de partícula (TP)

\begin{tabular}{|c|c|c|c|c|c|c|}
\hline Corante & $\begin{array}{l}\text { TP } \\
(\mu \mathrm{m})\end{array}$ & $\begin{array}{l}\mathrm{q}_{\exp } \\
\left(\mathrm{mg} \cdot \mathrm{g}^{-1}\right)\end{array}$ & $\mathrm{R}$ & $\begin{array}{l}\mathrm{k}_{2} \\
\left(\mathrm{~g} \cdot \mathrm{min}^{-1} \cdot \mathrm{mg}^{-1}\right)\end{array}$ & $\begin{array}{l}\mathrm{q}_{\mathrm{e}} \\
\left(\mathrm{mg} \cdot \mathrm{g}^{-1}\right)\end{array}$ & $\mathrm{h}\left(\mathrm{mg} \cdot \min ^{-1} \mathrm{~g}^{-1}\right)$ \\
\hline \multirow{3}{*}{$\begin{array}{l}\text { Azul de } \\
\text { metileno }\end{array}$} & 250 & 10,228 & 0,9716 & 0,0031 & 10,537 & 0,341 \\
\hline & 150 & 5,410 & 0,9962 & 0,0359 & 5,472 & 1,075 \\
\hline & 75 & 8,224 & 0,9967 & 0,0169 & 8,307 & 1,166 \\
\hline \multirow{3}{*}{$\begin{array}{l}\text { Verde } \\
\text { Malaquita }\end{array}$} & 250 & 7,425 & 0,9984 & 0,1430 & 9,987 & 6,980 \\
\hline & 150 & 7,316 & 0,9957 & 0,0140 & 7,457 & 0,790 \\
\hline & 75 & 8,426 & 0,9968 & 0,0942 & 8,697 & 7,130 \\
\hline
\end{tabular}

\subsection{Isoterma de adsorção}

Os experimentos foram conduzidos em batelada utilizando o tempo de equilíbrio de 360 minutos. As temperaturas foram $25,35,45$ e $65^{\circ} \mathrm{C} . \mathrm{O}$ ajuste ao modelo de Freundlich para a adsorção dos corantes azul de metileno e verde malaquita seguem na Tabela 3. 
Tabela 3-Parâmetros de ajuste ao modelo de Freundlich para adsorção dos corantes Azul de Metileno e Verde Malaquita

\begin{tabular}{c|c|c|c|c|c|c}
\hline \multirow{2}{*}{$\begin{array}{c}\text { Temperatura } \\
\left({ }^{\circ} \mathrm{C}\right)\end{array}$} & \multicolumn{3}{|c|}{ verde malaquita } & \multicolumn{3}{c}{ azul de metileno } \\
\cline { 2 - 7 } & $\mathrm{R}^{2}$ & $\begin{array}{c}\mathrm{K}_{\mathrm{F}} \\
\left(\mathrm{L} \cdot \mathrm{g}^{-1}\right)\end{array}$ & $\mathrm{N}$ & $\mathrm{R}^{2}$ & $\begin{array}{c}\mathrm{K}_{\mathrm{F}} \\
\left(\mathrm{L} . g^{-1}\right)\end{array}$ & $\mathrm{N}$ \\
\hline 25 & 0,90918 & 5,38791 & 1,04631 & 0,9886 & 4,0834 & 1,1137 \\
\hline 45 & 0,85014 & 5,03640 & 1,22435 & 0,8904 & 5,1784 & 1,1505 \\
\hline 65 & 0,94745 & 5,36822 & 0,78663 & 0,9612 & 4,5079 & 1,4040 \\
\hline
\end{tabular}

Comparando os valores dos coeficientes de correlação de ambos os modelos de Isotermas, observa-se que o modelo que melhor se adaptou aos dados experimentais foi o de Freundlich. Segundo Fritz e Schlünder (1981) omodelo de Freundlich considera a adsorção em multicamadas e é útil para descrever a adsorção em superfícies heterogêneas, como é o caso do adsorvente utilizado neste trabalho. Este modelo não considera as interações entre as moléculas, e é geralmente aplicado a adsorções físicas.

\subsection{Determinação dos parâmetros termodinâmicos}

Os parâmetros termodinâmicos estão na Tabela 4.Valores negativos para $\Delta \mathrm{G}$ indicam um processo espontâneo de adsorção. Ambos os corantes seguem um processo fisissortivo, pois apresentam valores de $\Delta \mathrm{H}$ menores que $40 \mathrm{~kJ} \mathrm{~mol}^{-1}$ e valores de $\Delta \mathrm{G}$ para a entre de 0 a $20 \mathrm{~kJ} . \mathrm{mol}^{-1}$ (Airoldi\& Faria, 2000; Sadasivam et al.; 2010).O valor negativo de $\Delta \mathrm{S}$ sugere que há uma diminuição no estado de desordem das moléculas durante o processo de adsorção, devido às interações com o adsorvente, com consequente diminuição de energia do meio.

Tabela 4 - Parâmetros Termodinâmicos

\begin{tabular}{|c|c|c|c|c|c|c|c|}
\hline \multirow[t]{2}{*}{ Corante } & \multirow{2}{*}{$\begin{array}{l}\mathrm{C}_{\mathrm{e}} \\
\left(\mathrm{mg} \cdot \mathrm{L}^{-1}\right)\end{array}$} & \multirow{2}{*}{$\begin{array}{l}\Delta \mathrm{H} \\
\left(\mathrm{kJ} \cdot \mathrm{mol}^{-1}\right)\end{array}$} & \multirow{2}{*}{$\begin{array}{c}\Delta \mathrm{S} \\
\left(\mathrm{J} \mathrm{mol}^{-1} \mathrm{~K}^{-1}\right)\end{array}$} & \multicolumn{4}{|c|}{$\Delta \mathrm{G}\left(\mathrm{kJ} \cdot \mathrm{mol}^{-1}\right)$} \\
\hline & & & & $298 \mathrm{~K}$ & $308 \mathrm{~K}$ & $318 \mathrm{~K}$ & $338 \mathrm{~K}$ \\
\hline Azul de Metileno & 3,0 & $-1,12883$ & $-2,09891$ & $-0,5697$ & & $-0,4997$ & $-0,4436$ \\
\hline Verde Malaquita & 3,0 & $-0,58508$ & $-0,41109$ & & $-0,4584$ & $-0,4543$ & $-0,4461$ \\
\hline
\end{tabular}

\subsection{Influência do pH}

Como observado na Figura 4 as melhores capacidades de adsorção para o corante azul de metileno foram nos valores de $\mathrm{pH}$ maiores que 6,0, já para o verde malaquita foram de 4,5 a 6,0 , valores estes que mostraram maior quantidade adsorvida por grama de adsorvente. Observa-se para ambos os corantes que a 3,5 a adsorção cai significativamente. Por outro lado, a pHs altos, os corantes comportam-se diferentemente: o verde malaquita mostra uma 
queda importante a $\mathrm{pH} 8$, enquanto que o azul de metileno continua sendo adsorvido a $\mathrm{pHs}$ maiores. Isto se deve às cargas contrárias da superfície do adsorvente e do adsorbato nos pontos de maior adsorção.

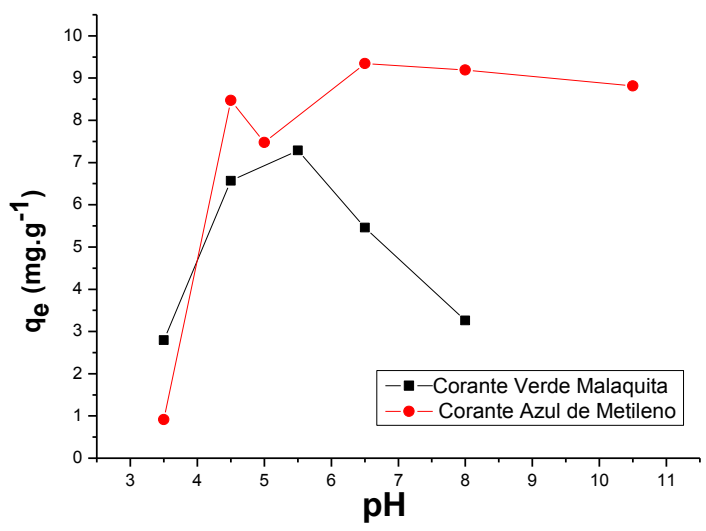

Figura 4. Capacidade de adsorção $\left(\mathrm{q}_{\mathrm{e}}\right)$ do verde malaquita e azul de metileno em diferentes $\mathrm{pHs}$.

\section{CONCLUSÃO}

Pode-se concluir neste trabalho que o talo de mandioca é um adsorvente viável na remoção dos corantes azul de metileno e verde malaquita, pois possui uma boa capacidade de adsorção, 8,426 mg.g ${ }^{-1}$ para o verde malaquita e $8,224 \mathrm{mg} \cdot \mathrm{g}^{-1}$ para o azul de metileno, para partícula de $75 \mu \mathrm{m}$ de diâmetro; além de possuir tempo de equilíbrio rápido, cerca de 6 horas para os dois corantes, e maior adsorção em $\mathrm{pH}$ próximo ao neutro. Os resultados encontrados neste trabalho caracterizam a adsorção dos corantes ácidos como um processo físico, o que torna a reversibilidade possível.

\section{REFERÊNCIAS BIBLIOGRAFICAS}

AIROLD, C.; FARIAS, R.F. O uso de sílica gel organofuncionalizada como agente sequestrante para metais. Química Nova, v. 23(4), (2000) 496-503.

BOEHM, H.P. Surface oxides on carbon and their analysis: a critical assessment, Carbon, 40, (2002) 145-149.

DISCHE, Z.; WHISTLER, R. L.; WOLFRAN, M. L. (ED.) .General color reactions. In:Carboydrates chemistry. New York: Academic Press. v.1, (1962) 477-512.

FARIA, P.C.C.; ÓRFÃO, J.J.M.; PEREIRA, M.F.R.. Adsorption of anionic and cationic dyes on activated carbons with different surface chemistries, Water Res. v.38 (2004) 2043-2052. 
FIORETTO, R.A. Uso direto da manipueira em fertirrigação. In CEREDA, M. P (Org). Manejo, uso e tratamento de subprodutos da industrialização de mandioca. São Paulo. Fundação Cargill, (2001). v 4, 320 p. (Série Culturas de Tuberosas Amiláceas Latino Americanas, v.4).

FRITZ, W.; SCHLÜNDER, E. U..Competivive adsorption of two dissolved organics onto activated cabon-I: Adsorption equilibria, Chem. Eng. Sci., v.36, (1981) 721-730.

GIDAMIS, A.B.; O’BRIEN, G.M. ; POULTER, N.H..Cassava detoxification of traditional Tanzanian foods.Int. J. Food Sci.,(1993) v. 28, p. 211-218.

INOUE, K. R. A.. Produção de Biogás, caracterização e aproveitamento agrícola do biofertilizante obtido na digestão da manipueira. Dissertação (Pós-graduação em Engenharia agrícola )- Universidade Federal de Viçosa, Minas gerais (2008) 4-9.

KOLPIN, D.W.; FURLONG, E.T.; MEYER, M.T.; THURMAN, E.M.; ZAUGG, S.D.; BARBER, L.B.; BUXTON, H.T..Pharmaceuticals, hormones, and other organic wastwater contaminants in U.S. Streams, 1999-2000: a national reconnaissance. Environmental Science Technology (2002) v. 36, n. 6, p. 1202-1211.

MAIAVES, A. H.. Caracterização Física, Físico Química e Potencial Tecnológico de novas Cultivares de Mandioca. Dissertação (Mestrado em Engenharia de Alimentos) - Universidade Federal de Santa Catarina, Florianópolis. (2010) 56-58.

PEREZ-MARIN, A.B.; MESEGUER ZAPATA, V.; ORTUNO, J.F.; AGUILAR, M.; SAEZ J.; LORÉNS, M.. Removal of cadmium from aqueous solutions by adsorption onto orange waste, J. Hazard.Mater. B139, (2007) 122-131.

SADASIVAM, S.; KRISHNA, S.K.; PONNUSAMY, K.; NAGARAJAN, G.S.; KANG, T.W., VENKATESALU, S.C..Equilibrium and thermodynamic studies on the adsorption of an organophosphorous pesticide onto "waste" jute fiber carbon.Journal of Chemical \& Engineering Data, v.55(12), (2010) 5658-5662.

SARASA, J.; ROCHE, M. P.; ORMAD, M. P.; GIMENO, E.; PUIG, A.; OVELLEIRO, J. L.. Treatment of a Wastewater Resulting from Dyes Manufacturing with Ozone and Chemical Coagulation.Water Research. (1998) v. 32, n. 9, p. 2721-2727.

TAPI .Preparation of Wood for chemical analysis,Technical Association of the Pulp and Paper Industry Association of Official Analytical Chemists (T 264 cm-97), ed. Tappi Press, Atlanta. (1997).

TAPI.Test methods,Technical Association of the Pulp and Paper Industry Association of Official Analytical Chemists (T 249 cm-85), Atlanta. (1999). 